\title{
OATY OF Mutations in MPNs: prognostic implications, window to biology, and impact on treatment decisions
}

\author{
Jamile M. Shammo ${ }^{1}$ and Brady L. Stein ${ }^{2}$ \\ ${ }^{1}$ Division of Hematology Oncology and Stem Cell Transplantation, Rush University Medical Center, Chicago, IL; \\ and ${ }^{2}$ Division of Hematology/Oncology and Robert H. Lurie Comprehensive Cancer Center, Department of \\ Medicine, Northwestern Feinberg University School of Medicine, Chicago, IL
}

The last decade has witnessed tremendous scientific advances, ushered in by the JAK2 V617F discovery, contributing to enhanced diagnostic capability and understanding of the biology of myeloproliferative neoplasms (MPNs). Discovery of the calreticulin mutations filled a diagnostic gap; more recent work sheds light on its contribution to disease pathogenesis, and prognosis. Recent studies have also identified novel JAK2 and MPL mutations in patients with essential thrombocythemia and myelofibrosis (MF). Especially in MF, the driver mutational profile has prognostic implications, with additive contributions from the acquisition of additional somatic mutations. The hope is that sophisticated molecular profiling will not only aid in prognostication, but also guide selection of therapy for patients with MPNs.

\section{Learning Objectives}

- To understand the biology and prognostic implications of currently identified mutations in MPNs

- To become familiar with literature outlining the impact of mutational profiling in the management of MPNs

\section{Introduction}

In the landmark perspective written by William Dameshek in 1951, the concept of "myeloproliferative disorders" as a related group of diseases was proposed. In this important paper, Dameshek alluded to the presence of a shared "myelostimulatory factor," which may have explained overlapping clinical and laboratory features in his "myeloproliferative disorders." the JAK2 V617F mutation was discovered in patients with essential thrombocythemia (ET), polycythemia vera (PV), and myelofibrosis (MF) ${ }^{2-5}$ Following this discovery, mutations of the thrombopoietin receptor (TPO-R) MPL were reported in a minority of ET and MF patients. ${ }^{6}$ In 2013, the first descriptions of the calreticulin $(C A L R)$ mutations were reported in patients with $J A K 2$ and $M P L$-negative ET or MF. ${ }^{7,8}$ These 3 mutations, referred from here on as "driver mutations" have clearly enhanced diagnostic capability. Recent reports, particularly focused on $C A L R$, have shed further light on contributions to disease pathogenesis. The driver mutational profile also influences prognosis, including vascular complication rates in ET and longevity in MF. Further, next-generation sequencing (NGS) techniques have allowed for a more nuanced understanding of prognosis. This review provides an overview of driver (JAK2/CALR/MPL) and other frequently reported mutations in myeloproliferative neoplasms (MPNs), discussing their prognostic and therapeutic implications, and role in routine clinical practice.

\section{Driver mutations \\ JAK2 mutations}

The JAK family of enzymes includes JAK1, JAK2, JAK3, and TYK2. These molecules attach to the cytosolic domains of cytokine receptors, and are essential for cytokine and growth factor signaling. JAK2 is the only member capable of mediating signaling through the 3 myeloid receptors (erythropoietin/MPL/granulocyte colonystimulating factor receptor).

The JAK2 V617F mutation is the result of a guanine to thymine change at nucleotide 1848 of exon 14 of the $J A K 2$ gene, which leads to a single amino acid substitution from valine to phenylalanine at codon 617 . The mutation results in dysregulated ligand-independent JAK2 kinase activity, due to its localization within the pseudokinase (JH2) domain, which negatively regulates activity of the kinase (JH1) domain. ${ }^{9} J A K 2$ V617F mediates the activation of downstream signaling through STATs (STAT5, STAT3, and STAT1), extracellular signal-regulated kinase/mitogen-activated protein kinase, and phosphatidylinositol 3-kinase/AKT/mammalian target of rapamycin pathways, resulting in uncontrolled myeloproliferation. Furthermore, JAK2 V617F has the capability to act as an epigenetic modifier, and has been reported to phosphorylate the protein arginine methyltransferase (PRMT5) with a much greater affinity than wildtype (WT) $J A K 2$, leading to a decreased methyltransferase activity and resultant myeloproliferation. ${ }^{10}$

Uniparental disomy at the $J A K 2$ locus on chromosome 9 results in homozygosity of the $J A K 2$ clone, and is responsible for the allelic variation in $J A K 2$ and phenotypic differences in $J A K 2$-driven MPNs. ${ }^{11}$

Recently, novel mutations of the JAK2 gene have been reported in a subset of patients with ET and primary myelofibrosis (PMF) who were negative for the JAK2 V617F mutation. ${ }^{12}$ It should be noted 
that the methods used to identify these mutations, such as whole exome sequencing (WES), are not currently available for adoption in routine daily practice.

\section{MPL mutations}

Located on chromosome 1p34, the MPL gene encodes for the TPO-R MPL, which signals through JAK2 and is considered essential for megakaryopoiesis. The first somatic MPL W515L mutation was described in 2006 , with a guanine to thymine change at nucleotide 1544 , resulting in a tryptophan to leucine substitution at codon $515 .{ }^{6}$ Several other gain-of-function mutations involving W515 have also been reported to occur in exon 10 of the $M P L$ gene, resulting in the substitution of tryptophan at codon 515 to lysine, arginine, or alanine, and corresponding to W515K, W515R, and W515A mutations, respectively. MPL mutations have been reported in $4 \%$ of patients with $\mathrm{ET}^{13}$ and $5 \%$ to $9 \%$ of patients with MF.,14

Novel MPL mutations had been recently identified by utilizing WES of granulocytes obtained from patients with ET and MF who lacked $J A K 2$ and MPL mutations. ${ }^{12,15}$ The ability to incorporate these novel mutations in diagnostic testing to demonstrate clonality is not yet feasible in clinical practice.

\section{CALR mutations}

CALR is a protein that resides in the lumen of the endoplasmic reticulum (ER), where it functions as a molecular chaperone for many glycoproteins, assisting in their folding and contributing to calcium homeostasis. CALR is also found outside of the ER where it has been implicated in a variety of biological processes, including proliferation and apoptosis. CALR has 3 main structural and functional domains: an $\mathrm{N}$-terminal lectin-binding domain, a proline-rich $\mathrm{P}$ domain, and a C-terminal acidic domain that contains multiple calcium-binding sites/KDEL necessary for binding to the ER. ${ }^{8,16}$ In 2013, 2 groups reported somatic mutations of the CALR gene, identified by exome sequencing of samples obtained from patients with $J A K 2$ V617F/MPL-negative ET or MF. Mutations were attributed to either base pair (bp) insertion or deletion on exon 9 of that gene, resulting in $\mathrm{a}+1 \mathrm{bp}$ frameshift, and the generation of a mutant protein with a novel C-terminus. ${ }^{7,8}$

Mutations in CALR are typically found in a heterozygous state with either a 52-bp deletion (type 1) or a 5-bp insertion (type 2 mutation) in the last exon encoding the C-terminal amino acid of the CALR protein, representing the most frequent types and found in $>80 \%$ of all patients with a $C A L R$-mutant MPN. ${ }^{17}$

All CALR genetic variants cause a loss of a sequence of 27 amino acids, leading to a loss of most of the C-terminal acidic domain and the KDEL sequence necessary for the function of the CALR protein and binding to the ER. ${ }^{7,8}$

The exact mechanism by which CALR mutations caused an MPN phenotype was not elucidated until recently, when 3 groups reported a novel signaling mechanism, whereby a mutant CALR protein constitutively activated receptor signaling through an abnormal interaction with the TPO-R (MPL). It was demonstrated that the interaction of CALR mutant proteins and the TPO-R directly led to dimerization and activation of JAK2 kinase. ${ }^{18-20}$ Interestingly, the extracellular domain of Tpo-R is reported to be essential for the TPOindependent activation by CALR mutant protein; however, additional studies are needed to determine the structural details of CALR mutants/TPO-R interaction. These data are particularly relevant because it was recently reported that TPO-R antagonists selectively deplete MF hematopoietic stem/progenitor cells and may represent a potentially new approach for treatment of MF patients. ${ }^{21}$

\section{Driver mutations and their prognostic implications JAK2 mutations and PV}

Because $\sim 95 \%$ of PV patients have $J A K 2 \mathrm{~V} 617 \mathrm{~F}$ mutations and 4\% harbor JAK2 exon 12 mutations, comparisons between JAK2 positive and negative patients are not plausible. The presence of $C A L R$ mutations in 2 cases of JAK2-negative PV has been reported, but this appears to be an exceptional circumstance. ${ }^{22}$ Although those with $J A K 2$ exon 12 mutations may have higher hemoglobin $(\mathrm{Hb})$, and lower platelet and leukocyte counts when compared with those with JAK2 V617F mutations, clinical outcomes do not differ, given similar incidences of thrombosis, MF, acute myeloid leukemia (AML), and death. ${ }^{23}$ However, associations between the JAK2 V617F allelic burden, clinical phenotype, and disease outcomes in PV patients have been reported. In particular, homozygous allele burden has been associated with older age, male sex, pruritus, and splenomegaly; associations between homozygous or increasing allelic burdens and thrombosis (arterial and venous), as well as MF transformation have also been suggested. ${ }^{24-29}$ Another well recognized phenotypic association with JAK2 V617F includes hepatic and portal vein thrombosis, but affected patients present a unique exception with regard to demographics, clinical phenotype, and allelic burden, because this complication is often observed in younger women, with either a masked phenotype, or lower leukocyte counts and lower allelic burdens. ${ }^{30,31}$ Mutations involving $M P L, J A K 2$ exon 12 , and $C A L R$ are infrequently identified relative to $J A K 2 \mathrm{~V} 617 \mathrm{~F}$ in patients presenting with abdominal vein thrombosis. ${ }^{30,32}$

\section{Driver mutations and ET}

In the initial descriptions, it was reported that $C A L R$-mutated ET patients had a lower $\mathrm{Hb}$ and leukocyte count, and a higher platelet count at diagnosis, compared with those with the JAK2 V617F mutation $(P<.0001){ }^{7}$ Further, CALR-mutated ET patients had a lower risk of thrombosis $(P=.003)$ and longer survival $(P=.04)$ compared with those with the $J A K 2 \mathrm{~V} 617 \mathrm{~F}$ mutation. ${ }^{7}$ Nangalia et al also observed higher platelet counts in $C A L R$-mutated ET patients, compared with those with $J A K 2$ mutations $(P<.001)$; however, although a higher rate of post-ET-MF transformation was reported in $C A L R$-mutated patients vs $J A K 2$-mutated patients $(P=.03)$, there were no differences in survival rates by mutation. ${ }^{8}$ Subsequently, large series have confirmed lower thrombosis rates in CALR-mutant ET patients, compared with those with $J A K 2$-mutant ET; interestingly, no $C A L R$-mutant ET patient evolved to PV, whereas the 10-year risk was near $30 \%$ in those with $J A K 2$-mutated $\mathrm{ET}^{33,34}$

Despite consistent reports of a lower thrombosis rate in CALRmutant ET patients, there appears to be little utility in incorporating CALR into the International Prognostic Score of Thrombosis in ET (IPSET)-thrombosis score, which places weight on the presence of the JAK2 V617F mutation. ${ }^{35}$ One possible explanation is the cosegregation of $C A L R$ mutations with other lower thrombotic risk features, such as younger age and less frequent history of thrombosis, which are already incorporated into the scoring system. ${ }^{36}$

Although the first descriptions of $C A L R$ mutations reported associations with longer survival ${ }^{7}$ or a higher rate of post-ET-MF transformation, ${ }^{8}$ these associations have been inconsistent. Perhaps, the associations may differ depending on the type of CALR 
mutation; a report of 908 ET patients suggested an association between type-1-like mutations and a higher rate of post-ET-MF transformation. ${ }^{37}$ In general, other large series have not been able to identify associations with MF and/or AML transformation or survival. $^{33,34}$ Although other large series with molecularly annotated ET patients identified a possible relationship between $M P L$ mutant-ET and post-ET-MF, there are no apparent differences in MF transformation rates among those with JAK2 and CALR mutations or in patients with no reported mutation; this is often referred to as triple-negative (TN) ET. ${ }^{38,39}$ These series noted that associations between mutational subgroup and survival were lost when adjusting for age distribution and gender. ${ }^{38,39}$

\section{Driver mutations and MF}

Initial descriptions of CALR mutations suggested that type 1 deletions were frequent in MF compared with ET; as in ET, MF patients with $C A L R$ mutations also had lower leukocyte counts and higher platelet counts compared with those with $J A K 2$-mutant MF. ${ }^{7}$ Longer overall survival was also noted in this initial study for those with $C A L R$-positive MF, compared with those with a JAK2 or MPL mutation. ${ }^{7}$

In a study of 617 patients, $64.7 \%$ carried $J A K 2$ V617F mutations, $22.7 \%$ had $C A L R$ mutations, $4 \%$ had MPL mutations, and $8.6 \%$ had a TN profile. ${ }^{17}$ Consistent with prior studies, at presentation, $C A L R$ mutated patients were younger, with lower leukocyte counts, higher platelet counts, and lower risk groupings; TN patients were older, with lower $\mathrm{Hb}$, lower platelet counts, and higher risk groupings. During the course of follow up, CALR-mutant patients had a lower cumulative incidence of developing anemia $(\mathrm{Hgb}<10 \mathrm{~g} / \mathrm{dL})$, thrombocytopenia $\left(<100 \times 10^{9} / \mathrm{L}\right)$, and marked leukocytosis $\left(>25 \times 10^{9} / \mathrm{L}\right)$, and a longer interval to the development of large splenomegaly ( $>10 \mathrm{~cm}$ below the left costal margin) compared with other mutational subgroups. ${ }^{17}$ Thrombosis rates were lower in those with CALR mutations $(13.6 \%)$ compared with those with $J A K 2$ V617F mutations $(18.3 \% ; P=.021)$. The 10 -year cumulative incidence of blast transformation was highest in TN patients (34.4\%), compared with those with JAK2 V617F $(19.4 \% ; P=.043$ for comparison), MPL (16.9\%), and CALR (9.4\%; $P=.016$ for comparison) mutations. Mutational profile also independently impacted median overall survival, which was 17.7 years for $C A L R$ mutated patients, 9.2 years for JAK2 V617F-mutated patients, 9.1 years for $M P L$-mutated patients, and 3.2 years for TN patients. When adjusting for age, $C A L R$-mutant patients still have improved overall survival compared with those with $J A K 2$ mutations or TN MF. ${ }^{17}$ The impact of the JAK2 V617F allelic burden was not examined in this study; previous findings have been inconsistent, although lower allelic burdens have been correlated with a worse outcome. $^{40}$

A study of 428 PMF patients also confirmed the prognostic impact of the driver mutational profile. ${ }^{38}$ Leukemia-free survival was worse in those with TN PMF compared with $C A L R, J A K 2$, and $M P L$-mutated MF; $C A L R$-mutated patients had a lower risk of blast transformation compared with those with TN and JAK2-mutated status. TN PMF patients also had the shortest median survival (2.3 years) compared with those with CALR (15.9 years), JAK2 (5.9 years), or MPL (9.9 years) mutations. Survival was better in patients with type $1 C A L R$ mutations compared with type $2 C A L R(P=.03)$ mutations or JAK2 $(P<.0001)$ mutations. ${ }^{38}$ Recently, a meta-analysis of 6 studies ( $\mathrm{n}=1381$ ), including a diverse PMF patient population, confirmed the prognostic impact of $C A L R$ with improved survival compared with those with JAK2 mutations, but only in the non-Asian population. ${ }^{41}$ It was hypothesized that the lack of survival benefit in Asian patients could be due to a higher prevalence of type 2 CALR mutations in Asian patients. ${ }^{41}$ In keeping with studies of this rare population, the differential impact on MF outcomes between type 1 and type $2 C A L R$ mutations has been inconsistent; a recent study could not identify differences in overall survival in those with type-1-like vs type-2-like CALR mutations. ${ }^{37}$

Interestingly, the driver mutational profile has less impact on prognosis in those with secondary MF, based on a study of 359 patients with post-PV-MF $(\mathrm{n}=194)$ and post-ET-MF $(\mathrm{n}=165){ }^{29}$ Only TN post-ET-MF had a shorter survival compared with $C A L R$ mutated post-ET-MF $(P=.01)$, and there was no difference between other genotypes, including type 1 vs type $2 C A L R, J A K 2$, and $M P L$-mutated secondary $\mathrm{MF}^{29}$

\section{Other somatic mutations in MPNs and their prognostic implications}

Recent utilization of NGS has allowed for simultaneous profiling of multiple genes and has led to the identification of novel somatic mutations in patients with a variety of myeloid neoplasms, including MPNs, mostly occurring in patients with MF (Table 1). ${ }^{2-8,42-50}$ These somatic mutations can involve genes in the spliceosome machinery (SF3B1, U2AF1, and SRSF2), as well as genes encoding for several epigenetic modifiers (TET2, DNMT3A, IDH1/2, EZH2, and ASXL1). Even though reported somatic mutations lack specificity, because they can be found in a broad range of myeloid neoplasms, there is evidence to suggest that the identification of certain nondriver mutations in MPN patients is associated with greater risk of disease progression or shortened survival.

\section{Other somatic mutations in ET and PV}

Genetic complexity extends beyond the presence of driver mutations in ET and PV. WES of $48 \mathrm{PV}$ and 62 ET samples identified a median of 6.5 mutations per patient in each group (compared with 13 in $\mathrm{MF}){ }^{8}$ Albeit at low frequencies, in PV, the most commonly mutated, nondriver genes included TET2, followed by DNMT3A. In ET, the most commonly mutated, nondriver genes, also at low frequencies, included DNMT3A, TET2, and ASXL1. ${ }^{8}$ Interestingly, the order in which mutations are acquired appears to influence clinical features. Through genotyping hematopoietic colonies or using NGS, it was suggested that those patients who acquired JAK2 V617F prior to TET2 were younger, more likely to present with PV than ET, and had higher thrombosis rates, when compared with patients who acquired TET2 mutations prior to JAK2 V617F. ${ }^{51}$ This study also suggested that mutant progenitors from "JAK2-first" patients were more sensitive to JAK inhibition compared with "TET2-first" patients. ${ }^{51}$

Recently, the prevalence and relevance of somatic mutations was reported in a different cohort, including $133 \mathrm{PV}$ and $181 \mathrm{ET}$ patients. ${ }^{52}$ In PV, $44 \%$ were reported to have mutations, including $29 \%$ with 1 mutation, $14 \%$ with 2 mutations, and $1 \%$ with 3 mutations. The most common mutations involved TET2 (18\%), ASXL1 (11\%), $S H 2 B 3(5 \%)$, and SF3B1 (3\%). The number of mutations impacted overall and MF-free survival; and the hazard ratio (HR) was 2.6 and 13.7, respectively, for those with 2 mutations, compared with 1.7 and 5.1 for those with 1 mutation. In a multivariable analysis, SRSF2 and RUNX1 affected overall survival, IDH2 and RUNX1 impacted leukemia-free survival, and ASXL1, IDH2, RUNX1, KIT, and $S E T B P 1$ predicted fibrotic progression. ${ }^{52}$ In ET, $46 \%$ of patients 
Table 1. Frequency of molecular mutations in classical MPNs

\begin{tabular}{|c|c|c|c|c|c|}
\hline Affected pathways & Mutation & PMF (\%) & PV (\%) & ET (\%) & Localization/relevance \\
\hline \multirow[t]{6}{*}{ Cytokine signaling } & $J A K 2 V 617 F$ & $50-60$ & 95 & $50-60$ & $\begin{array}{l}\text { Chromosome 9p24. Most frequent gain-of-function } \\
\text { mutation in } \mathrm{PV}^{2-5}\end{array}$ \\
\hline & $J A K 2$ exon 12 & - & $3-4$ & - & $\begin{array}{l}\text { A gain-of-function mutation, although outside the auto- } \\
\text { inhibitory domain of JAK2. Not found in ET/PMF, can be } \\
\text { present in post-PV-MF }\end{array}$ \\
\hline & $M P L$ & 9 & - & 4 & Chromosome 1p34. A gain-of-function mutation ${ }^{6}$ \\
\hline & CALR & $20-25$ & - & $20-25$ & $\begin{array}{l}\text { Chromosome } 19 \mathrm{p} 13.2^{7,8} \\
\text { All described mutations are either indels in the last exon } \\
\text { encoding for the C-terminal amino acid of CALR protein } \\
\text { (type 1: } 52 \text { bp deletion; type } 2: 5 \text { bp insertion are the } \\
\text { most common), resulting in a mutant protein with loss of } \\
\text { ER retention signal }\end{array}$ \\
\hline & $C B L$ & 6 & - & Rare & $\begin{array}{l}\text { Chromosome 11q23.3. A loss-of-function mutation with } \\
\text { loss of inhibition of cytokine signaling due to abrogated } \\
\text { CBL ubiquitin ligase activity }{ }^{42}\end{array}$ \\
\hline & LNK & Rare & Rare & Rare & $\begin{array}{l}\text { Chromosome } 12 \mathrm{p} 24 \text {. A loss-of-function mutation with } \\
\text { resultant loss of LNK-associated negative regulation of } \\
\text { cytokine receptor signaling }\end{array}$ \\
\hline \multirow[t]{2}{*}{ Spliceosomes } & SRSF2 & 17 & - & - & $\begin{array}{l}\text { SRSF2 mutations are relatively common in PMF, cluster } \\
\text { with IDH mutations, and are independently predictive of } \\
\text { poor outcome }\end{array}$ \\
\hline & SF3B1 & 6.5 & - & Rare & $\begin{array}{l}\text { A spliceosome mutation. Mutually exclusive of other } \\
\text { spliceosomal mutations }{ }^{49}\end{array}$ \\
\hline \multirow[t]{4}{*}{ Epigenetic modifiers } & $A S X L 1$ & $8-26$ & 2 & Rare & $\begin{array}{l}\text { Chromosome } 20 \mathrm{q} 11.21 \text {. ASXL1 encodes a transcription } \\
\text { factor, which functions through histone modification. } \\
\text { Mutations affecting exon } 12 \text { are found mostly in } \\
\text { PMF }^{47,48}\end{array}$ \\
\hline & $I D H 1 / 2$ & 4.2 & 1.9 & 0.8 & $\begin{array}{l}\text { Chromosomes } 2 q 33.3 / 15 q 26.1 \text {. Mutants cause } \\
\text { overproduction of 2-hydroxyglutarate, which inhibits } \\
\text { TET2/other KG-dependent enzymes. }{ }^{43} \text { Presence of } \\
\text { mutation may be explored therapeutically, similar to the } \\
\text { ongoing IDH inhibitor studies in AML }\end{array}$ \\
\hline & $E Z H 2$ & 13 & 3 & - & $\begin{array}{l}\text { Chromosome } 7 q 35 \text {. Mutations lead to loss of epigenetic } \\
\text { regulation, and are typically associated with poor } \\
\text { outcome in } \mathrm{PMF}^{46}\end{array}$ \\
\hline & TET2 & 8 & 10 & 5 & $\begin{array}{l}\text { Chromosome } 4 \text { q24. Loss-of-function mutations resulting } \\
\text { in decreased } 5 \text {-hydroxymethylcytosine, and interfering } \\
\text { with cytosine demethylation. }{ }^{45} \text { TET2 mutation may have } \\
\text { an impact on ET vs PV phenotype (see text) }\end{array}$ \\
\hline
\end{tabular}

$\mathrm{IDH}$, isocitrate dehydrogenase; Indels, insertions and deletions; KG, ketoglutarate.

had somatic mutations; the most common mutations involved TET2 (13\%), ASXL1 (11\%), DNMT3A (6\%), SF3B1 (5\%), CEBPA (4\%), and TP53, SH2B3,EZH2, and CSF3R (2\% each). The number of mutations (HR, 6.6 for 3 mutations and HR, 2.2 for 1 or 2 mutations) impacted overall survival, but not leukemia or MFfree survival. In multivariable analysis, EZH2 and $S F 3 B 1 \mathrm{mu}-$ tations impacted survival. ${ }^{52}$

At present, there is little evidence to suggest the incorporation of testing for nondriver mutation in routine clinical care of patients with ET or PV.

\section{Other somatic mutations and their prognostic implications in MF}

In order to clarify the prognostic relevance of nondriver mutations and their impact on survival, an international collaborative project analyzed the outcome of 879 patients with PMF and known mutational status. ${ }^{53}$ ASXL1 mutations correlated with constitutional symptoms, leukocytosis, and $\geq 1 \%$ circulating blasts; SRSF 2 mutations correlated with older age; and EZH2 mutations associated with $\geq 1 \%$ circulating blasts. Patients with $A S X L 1, E Z H 2$, SRSF2, or $I D H$ mutations were at risk for premature death or leukemic transformation. Patients with any one of these mutations are considered to have a "high molecular risk" profile (HMR). However, only ASXL1 mutations remained significantly associated with survival in the context of the International Prognostic Scoring System (IPSS) ${ }^{53}$ Interestingly, it was subsequently demonstrated that the number of mutations also matters, because the presence of 2 or more mutations predicted for worse outcomes; the reported median survival was 12.3 years for patients without a mutation compared with 2.6 years for those with 2 or more mutations. ${ }^{54}$

Another report on comprehensive mutational screening of 104 genes by NGS at diagnosis and during follow up $(\mathrm{N}=197)$ demonstrated the presence of somatic mutations in $90 \%$ of patients, and $37 \%$ carried somatic mutations other than JAK2 V617F or CALR. The presence of $\geq 2$ somatic mutations significantly reduced overall survival and increased the risk of AML transformation. Somatic mutations with loss of heterozygosity in TP53 were strongly associated with leukemic transformation. ${ }^{55}$ 
A

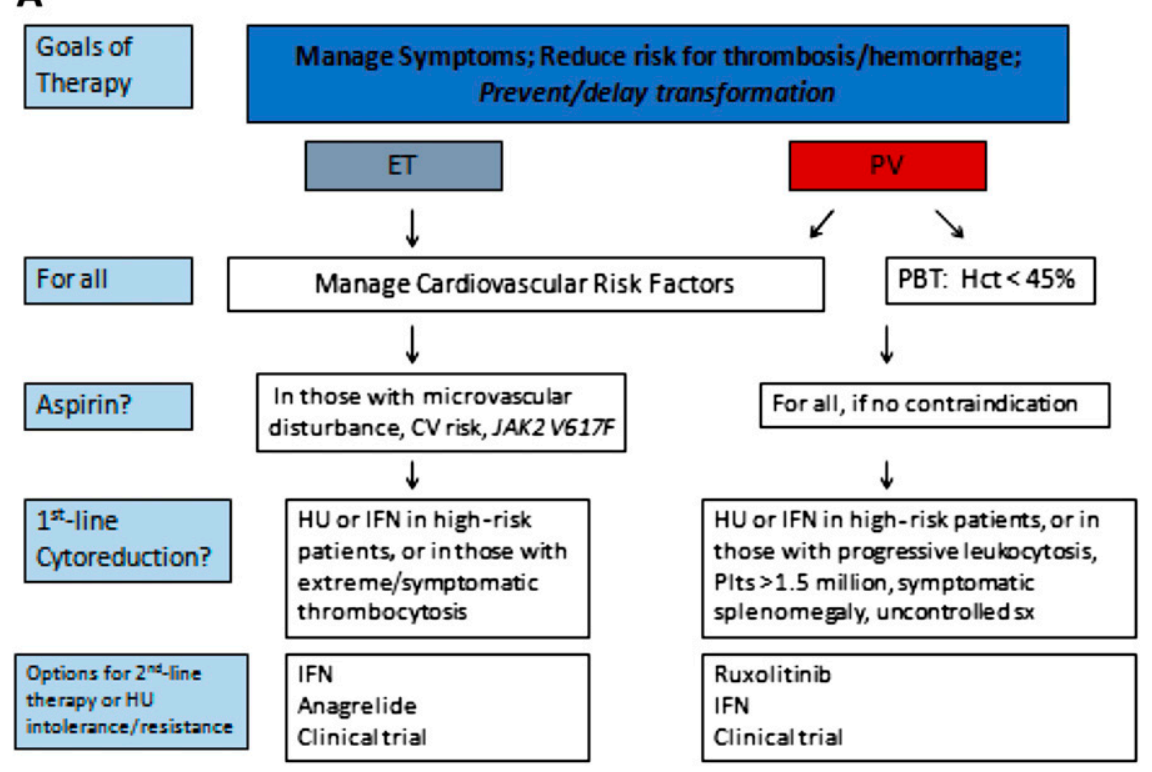

B

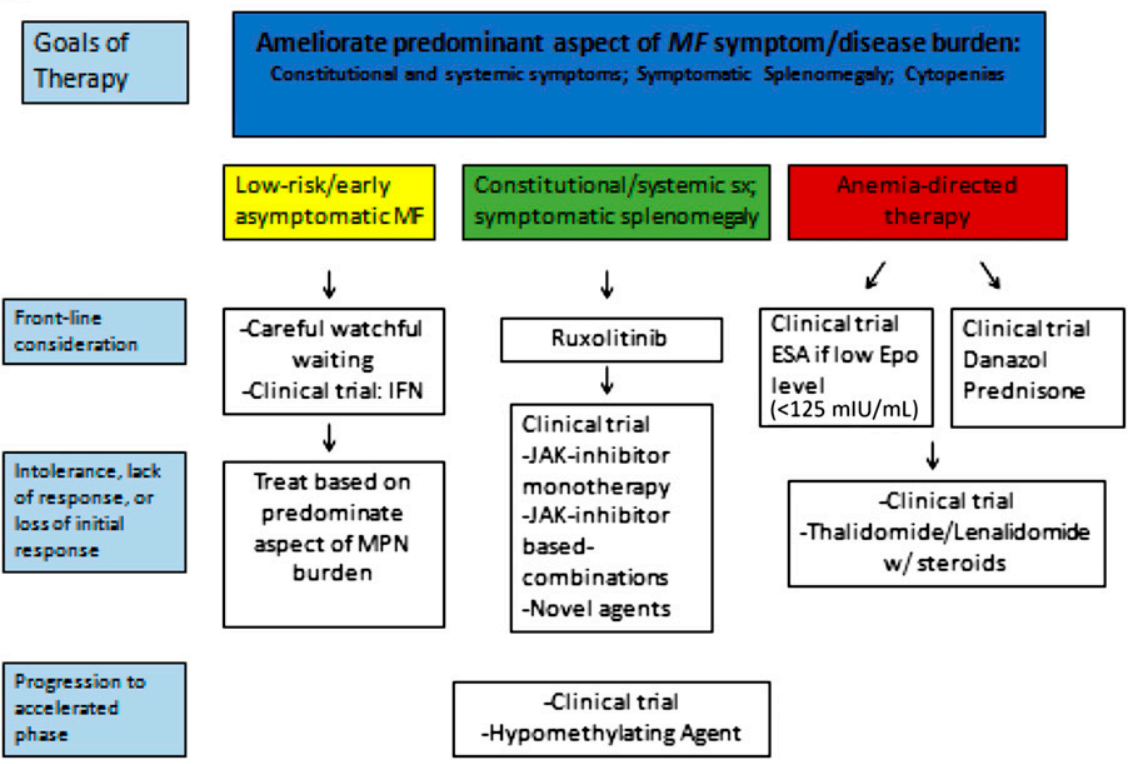

Figure 1. (A) How we approach the management of ET and PV. (B) How we approach nontransplant management of MF. CV, cardiovascular; Epo, erythropoietin; ESA, erythropoiesis-stimulating agent; Hct, hematocrit; HU, hydroxyurea; PBT, phlebotomy; Plts, platelets; symptoms (bone pain, fever, night sweats, and weight loss).

The impact of the mutational profile on prognosis has been reported. ${ }^{56}$ Among 570 patients, those $C A L R$-mutated/ASXL1negative patients had a median 10.4-year survival, $C A L R$-mutated/ $A S X L 1$-positive or $C A L R$-negative/ASXL1-negative patients had a median 5.8-year survival, and CALR-negative/ASXL1-positive patients had a median 2.3-year survival. ${ }^{56}$ Extending on this theme, new prognostic scoring systems incorporating molecular and genetic features of MF have been proposed. ${ }^{57,58}$

As previously mentioned, the impact of the mutational profile differs in those with secondary MF, including post-ET and post-PV-MF. ${ }^{29}$ Those with post-ET-MF were more likely to have $A S X L 1$ and $E Z H 2$ mutations, compared with those with post-PV-MF (29\% vs $17 \%$,
$P=.011$ and 10.3 vs $3.6 \%, P=.022$, respectively). However, in post-PV-MF, there was no association between a single-somatic gene mutation, HMR profile, or number of HMR-mutations and overall survival. In post-ET-MF, only $S R S F 2$-mutated status correlated with shortened survival $(P=.001 ; 4.9$ years vs 14.5 years; mutated vs WT).

\section{Molecular mutations and therapeutic implications}

Treatment decisions for MPN patients are not yet driven by the presence or absence of MPN-associated molecular mutations, but rather, influenced by MPN subtype, symptom burden, and risk category (Figure 1). Importantly, risk classifications are evolving, and the influence of mutations is increasingly clear (Figure 2). ${ }^{57,58}$ 

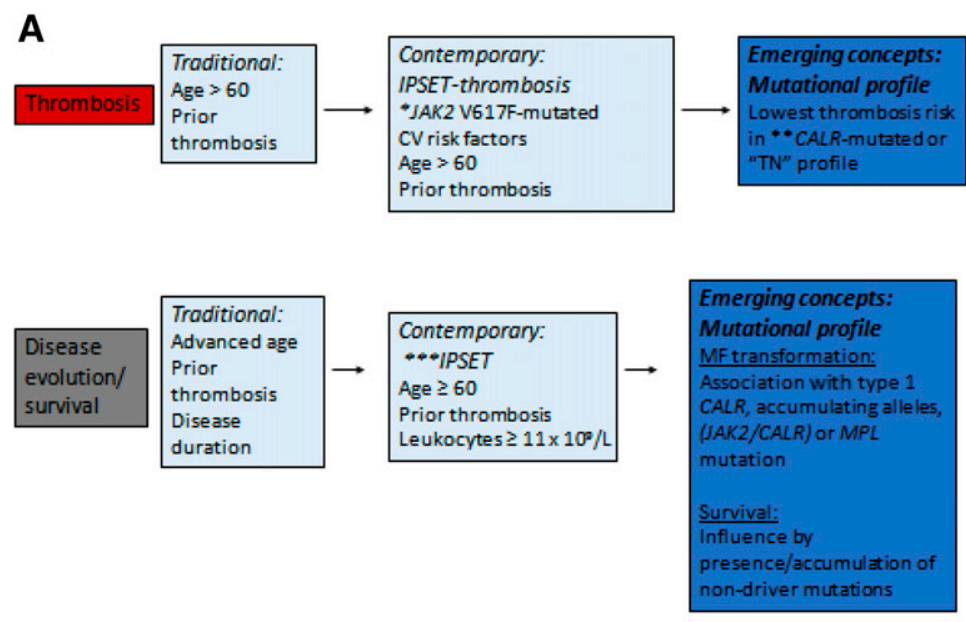

B
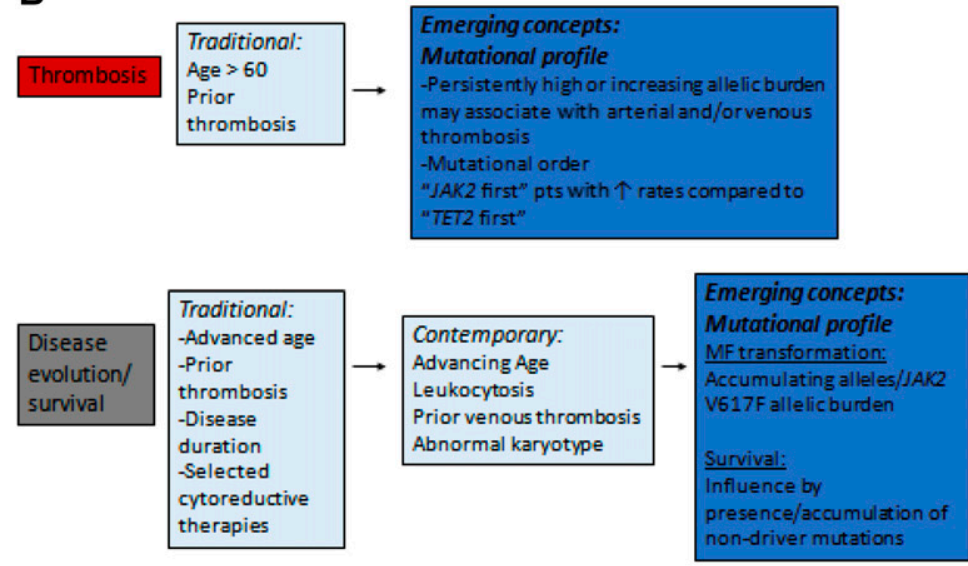

C

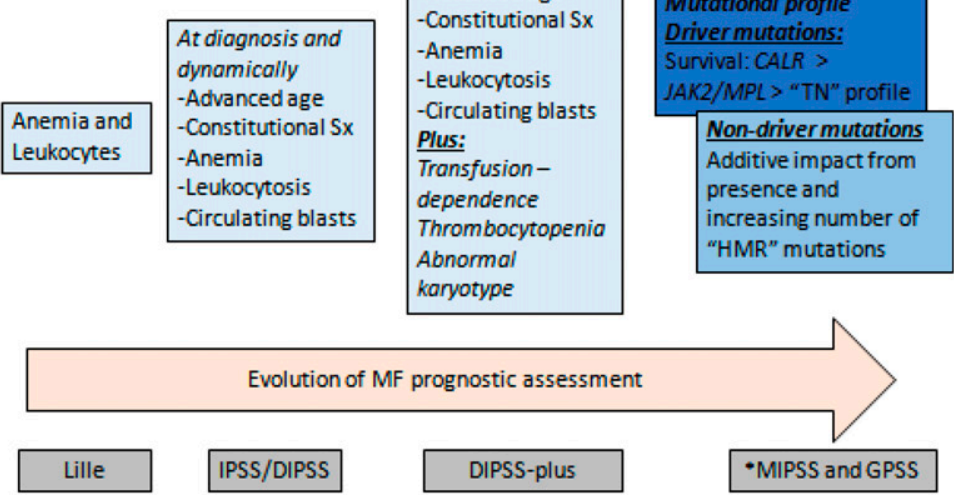

Figure 2. (A) Evolution in prognostic assessment: ET. Constructed prior to CALR discovery $\left.{ }^{*}\right)$. CALR co-segregates with younger age and absent thrombosis risk, and therefore, does not modify IPSET score $\left.{ }^{* \star}\right)$. Unable to predict MF or AML risk $\left.{ }^{\star \star \star \star}\right)$. (B) Evolution in prognostic assessment: PV. (C) Evolution in prognostic assessment: MF. The latest proposed MF scoring systems incorporate molecular and genetic information in the assessment $\left(^{*}\right)$. The GPSS identified very high (3 points) and high-risk karyotypes (2 points), TN status (2 points), JAK2/MPL-mutated (2 points), type-2/type-2-like CALR-mutated (2 points), ASXL 1-mutated (1 point), and SRSF2-mutated (1 point), as independent predictors of shortened survival; these variables were included in this score, along with age $>60$ years. ${ }^{57}$ Another system, the Mutation-enhanced IPSS (MIPSS), analyzed 986 PMF patients, identifying age $>60$, constitutional symptoms, $\mathrm{Hb}<10 \mathrm{~g} / \mathrm{dL}$, platelets $<200 \times 10^{9} / \mathrm{L}$, TN status (1.5 points), JAK2-mutated or MPL-mutated (0.5 points), ASXL1mutated (0.5 points), and SRSF2-mutated (0.5 points) status as significant, adverse indicators. ${ }^{58} \mathrm{CV}$, cardiovascular; DIPSS, dynamic IPSS; GPSS, genetics-based Prognostic Scoring System; pts, patients. 
Hematopoietic stem cell transplant represents the only curative treatment option in MF, but is typically reserved for patients with intermediate-2 or high-risk disease (noting the impact that somatic mutations may have), and will be discussed in detail elsewhere (see Gupta, in this book $^{59}$ ).

\section{Mutations and response to therapy in ET and PV}

In ET and PV, there has been renewed interest in using pegylatedinterferon (IFN), and pivotal clinical trials are underway to elucidate its role in the upfront treatment of such patients (\#NCT01259856 and \#NCT01259817). In part, intrigue is related to not only high rates of hematologic responses, but also molecular responses initially reported in JAK2-mutated ET and PV patients. In a phase 2 trial, with a median of 42 months follow up, complete hematologic responses (CHR) and complete molecular responses (CMR) were seen in $76 \%$ (18\% CMR) of PV and 77\% (17\% CMR) of ET patients. ${ }^{60}$ In patients with $\mathrm{PV}$, there was a sustained decrease in the allelic burden, with a decrease from a median of $64 \%$ to $8 \%$ in those treated for 60 months. In this study, the presence of somatic mutations impacted outcomes, because a higher proportion of patients who did not achieve CMR (56\% vs 30\% in those with CMR) had mutations (most commonly, TET2, DNMT3A, and ASXL1) ${ }^{60}$ Further, in those with paired samples, clonal evolution was commonly observed in those who failed to achieve CMR. Finally, those with JAK2/TET2 mutations had higher $J A K 2$ allelic burdens, and less significant reductions during the course of therapy compared with JAK2mutant/TET2-WT patients. ${ }^{60}$ Interestingly, a molecular response does not always accompany a hematologic response in $\mathrm{PV}{ }^{61}$

Responses to IFN have also been recently reported in $C A L R$-mutated ET patients. ${ }^{62}$ Among 31 patients, $77 \%$ achieved a CHR; the median CALR burden was $41 \%$ and a molecular response rate of $42 \%$ was reported (CMR plus partial MR). In a cohort of CALR-ET patients treated with hydroxyurea or aspirin, the $C A L R$ burden remained stable. $^{62}$ This study also analyzed the impact of somatic mutations, and demonstrated that those with additional mutations had poorer molecular responses compared with those with $C A L R$ alone. Analyses of mutation type also suggested a differential effect from IFN- $\alpha$ on mutated clones, because some patients experienced a decrease in the $C A L R$ burden but an increase in the clonal burden of other somatic mutations. ${ }^{62}$

Another novel ET treatment concept involves telomerase inhibition. Among 18 previously treated patients, $89 \%$ had a CHR. ${ }^{63}$ Responses were seen regardless of the mutational profile, although more pronounced in those with $J A K 2$ mutations, as molecular partial response rates of $88 \%$ were reported in this group; mutant allele burdens were also reduced by $15 \%$ to $66 \%$ in those with $M P L$ or $C A L R$ mutations. $^{63}$

\section{Mutations and response to therapy in MF}

Whereas the driver mutational profile influences prognosis, there is less impact on response to JAK inhibition. In the phase 3 studies of ruxolitinib compared with placebo or best available therapy, there was no statistically significant difference in efficacy measures when comparing JAK2-mutated and WT patients. ${ }^{64,65}$ Subsequently, a letter reported on spleen and symptom responses to the JAK inhibitor, fedratrinib, in patients with $C A L R$-mutated $M F .^{66}$ The clinical observation of JAK-inhibitor response regardless of mutational profile is supported by recent translational studies revealing an activated JAK2 signaling signature in MPN patients irrespective of mutational profile. $^{67}$
Studies have subsequently examined the potential impact of somatic mutations on response to MF therapy. Using data from the COMFORT-II study, ${ }^{65}$ ruxolitinib-treated patients with HMR profiles (presence of $A S X L 1, E Z H 2, S R S F 2$, or $I D H$ mutations) were compared with those with low-molecular risk profiles (defined as absence of the aforementioned mutations). Interestingly, there was no difference in rates of spleen volume reduction or symptom relief; further, similar rates of hematologic toxicity were seen in both groups. The investigators also reported improved survival, regardless of mutational profile, with reduction in the risk of death even in those ruxolitinib-treated patients with HMR profiles compared with best therapy $(\mathrm{HR}, 0.57)$. Another study of patients treated with ruxolitinib reported similar spleen responses in patients regardless of $J A K 2, C A L R, M P L$, or TN status, but those with 1 or more mutations in $A S X L 1, E Z H 2$, or $I D H 1 / 2$ were less likely to have a spleen response. ${ }^{68}$ The number of mutations inversely correlated with spleen response and time to treatment discontinuation, with the worst outcomes observed in those with $\geq 3$ mutations. ${ }^{68}$

A study of imetelstat presents an exception to the observations of efficacy irrespective of the MF-driver mutational status. In a pilot study of 33 patients, a complete or partial response was reported in $21 \%$; although not statistically significant, and unlike the study in ET, response rates were $27 \%$ in those with the JAK2 mutation vs $0 \%$ in those without the $J A K 2$ mutation $(P=.3){ }^{69}$ Other somatic mutations also impacted response rates, because $32 \%$ without an ASXL1 mutation responded, compared with $0 \%(P=.07)$ among those with an $A S X L 1$ mutation. Those with mutations of $S F 3 B 1$ or $U 2 A F 1$ had the highest complete response rates of $38 \%$, compared with $4 \%$ in those without these mutations $(P=.04){ }^{69}$

\section{Mutational profiles in clinical practice and the future}

It is clear that the driver mutational profile complements the diagnostic approach to MPNs, as discussed. In ET, the presence of $C A L R$ typically co-segregates with younger age and absence of a thrombosis history; in this way, perhaps identifying patients at lower risk for vascular complications. The impact of the driver mutational profile on prognosis appears strongest in MF, helping set expectations in the clinic; some MF patients with CALR mutations may enjoy a period of longevity. The availability of several NGSbased myeloid panels from commercial and academic laboratories begets the question as to whether they should be used and incorporated in clinical practice. Accruing evidence suggests that identification of certain somatic mutations provide important prognostic information, particularly in MF. The presence of these mutations may upgrade the prognosis to a higher than expected risk category, which may aid in making treatment recommendations, particularly when considering stem cell transplantation. Ideally, a prospective evaluation of mutational profiles would identify patterns of acquisition of new mutations with disease progression and clonal evolution, assess impact of disease duration and therapy on development/evolution of these mutations, and help clinicians in determining whether or not monitoring allele variance frequency is necessary for all myeloid neoplasm patients. In today's routine clinical practice, however, NGS will not yet guide routine therapeutic decision making. Use of NGS in ET/PV is less established, and in the authors' opinion, the results are even less likely to be actionable. Because most panels have the capacity to test multiple genes, they can identify somatic mutations involving signaling molecules, epigenetic regulators, tumor suppressor genes, transcription factors, and splicing factors. Ideally, the identification of such molecular signatures or specific pathway aberrancies will lead to the conduct of 
rational clinical trials involving novel, precise, and targeted therapies. Nonetheless, a nuanced awareness of Dameshek's "myelostimulatory factors" signifies a breakthrough in understanding for physicians and scientists, and hopefully changes the course for MPN patients.

\section{Correspondence}

Jamile M. Shammo, Section of Hematology, Rush University Medical Center, 1725 W. Harrison St, Suite 809, Chicago IL 60612; e-mail: jamile_shammo@rush.edu.

\section{References}

1. Dameshek W. Some speculations on the myeloproliferative syndromes. Blood. 1951;6(4):372-375.

2. Baxter EJ, Scott LM, Campbell PJ, et al; Cancer Genome Project. Acquired mutation of the tyrosine kinase JAK2 in human myeloproliferative disorders. Lancet. 2005;365(9464):1054-1061.

3. James C, Ugo V, Le Couédic JP, et al. A unique clonal JAK2 mutation leading to constitutive signalling causes polycythaemia vera. Nature. 2005;434(7037):1144-1148.

4. Kralovics R, Passamonti F, Buser AS, et al. A gain-of-function mutation of JAK2 in myeloproliferative disorders. $N$ Engl J Med. 2005;352(17): 1779-1790.

5. Levine RL, Wadleigh M, Cools J, et al. Activating mutation in the tyrosine kinase JAK2 in polycythemia vera, essential thrombocythemia, and myeloid metaplasia with myelofibrosis. Cancer Cell. 2005;7(4): 387-397.

6. Pikman Y, Lee BH, Mercher T, et al. MPLW515L is a novel somatic activating mutation in myelofibrosis with myeloid metaplasia. PLoS Med. 2006;3(7):e270.

7. Klampfl T, Gisslinger H, Harutyunyan AS, et al. Somatic mutations of calreticulin in myeloproliferative neoplasms. N Engl J Med. 2013; 369(25):2379-2390.

8. Nangalia J, Massie CE, Baxter EJ, et al. Somatic CALR mutations in myeloproliferative neoplasms with nonmutated JAK2. $N$ Engl J Med. 2013;369(25):2391-2405.

9. Saharinen P, Silvennoinen O. The pseudokinase domain is required for suppression of basal activity of Jak2 and Jak3 tyrosine kinases and for cytokine-inducible activation of signal transduction. J Biol Chem. 2002; 277(49):47954-47963.

10. Liu F, Zhao X, Perna F, et al. JAK2V617F-mediated phosphorylation of PRMT5 downregulates its methyltransferase activity and promotes myeloproliferation. Cancer Cell. 2011;19(2):283-294.

11. Scott LM, Scott MA, Campbell PJ, Green AR. Progenitors homozygous for the V617F mutation occur in most patients with polycythemia vera, but not essential thrombocythemia. Blood. 2006;108(7):2435-2437.

12. Milosevic Feenstra JD, Nivarthi H, Gisslinger H, et al. Whole-exome sequencing identifies novel MPL and JAK2 mutations in triple-negative myeloproliferative neoplasms. Blood. 2016;127(3):325-332.

13. Rumi E, Pietra D, Guglielmelli P, et al; Associazione Italiana per la Ricerca sul Cancro Gruppo Italiano Malattie Mieloproliferative. Acquired copy-neutral loss of heterozygosity of chromosome $1 \mathrm{p}$ as a molecular event associated with marrow fibrosis in MPL-mutated myeloproliferative neoplasms. Blood. 2013;121(21):4388-4395.

14. Pardanani AD, Levine RL, Lasho T, et al. MPL515 mutations in myeloproliferative and other myeloid disorders: a study of 1182 patients. Blood. 2006;108(10):3472-3476.

15. Cabagnols X, Favale F, Pasquier F, et al. Presence of atypical thrombopoietin receptor (MPL) mutations in triple-negative essential thrombocythemia patients. Blood. 2016;127(3):333-342.

16. Wang WA, Groenendyk J, Michalak M. Calreticulin signaling in health and disease. Int J Biochem Cell Biol. 2012;44(6):842-846.

17. Rumi E, Pietra D, Pascutto C, et al; Associazione Italiana per la Ricerca sul Cancro Gruppo Italiano Malattie Mieloproliferative Investigators. Clinical effect of driver mutations of JAK2, CALR, or MPL in primary myelofibrosis. Blood. 2014;124(7):1062-1069.
18. Marty $\mathrm{C}$, Pecquet $\mathrm{C}$, Nivarthi $\mathrm{H}$, et al. Calreticulin mutants in mice induce an MPL-dependent thrombocytosis with frequent progression to myelofibrosis. Blood. 2016;127(10):1317-1324.

19. Chachoua I, Pecquet C, El-Khoury M, et al. Thrombopoietin receptor activation by myeloproliferative neoplasm associated calreticulin mutants. Blood. 2016;127(10):1325-1335.

20. Araki M, Yang Y, Masubuchi N, et al. Activation of the thrombopoietin receptor by mutant calreticulin in CALR-mutant myeloproliferative neoplasms. Blood. 2016;127(10):1307-1316.

21. Wang X, Haylock D, Hu CS, et al. A thrombopoietin receptor antagonist is capable of depleting myelofibrosis hematopoietic stem and progenitor cells. Blood. 2016;127(26):3398-3409.

22. Broséus J, Park JH, Carillo S, Hermouet S, Girodon F. Presence of calreticulin mutations in JAK2-negative polycythemia vera. Blood. 2014; 124(26):3964-3966

23. Passamonti F, Elena C, Schnittger S, et al. Molecular and clinical features of the myeloproliferative neoplasm associated with JAK2 exon 12 mutations. Blood. 2011;117(10):2813-2816.

24. Godfrey AL, Chen E, Pagano F, Silber Y, Campbell PJ, Green AR. Clonal analyses reveal associations of JAK2V617F homozygosity with hematologic features, age and gender in polycythemia vera and essential thrombocythemia. Haematologica. 2013;98(5):718-721.

25. Passamonti F, Rumi E, Pietra D, et al. A prospective study of 338 patients with polycythemia vera: the impact of JAK2 (V617F) allele burden and leukocytosis on fibrotic or leukemic disease transformation and vascular complications. Leukemia. 2010;24(9):1574-1579.

26. Vannucchi AM, Antonioli E, Guglielmelli P, et al; MPD Research Consortium. Prospective identification of high-risk polycythemia vera patients based on JAK2(V617F) allele burden. Leukemia. 2007;21(9):1952-1959.

27. Borowczyk M, Wojtaszewska M, Lewandowski K, et al. The JAK2 V617F mutational status and allele burden may be related with the risk of venous thromboembolic events in patients with Philadelphia-negative myeloproliferative neoplasms. Thromb Res. 2015;135(2):272-280.

28. Alvarez-Larrán A, Bellosillo B, Pereira A, et al. JAK2V617F monitoring in polycythemia vera and essential thrombocythemia: clinical usefulness for predicting myelofibrotic transformation and thrombotic events. Am J Hematol. 2014;89(5):517-523.

29. Rotunno G, Pacilli A, Artusi V, et al. Epidemiology and clinical relevance of mutations in postpolycythemia vera and postessential thrombocythemia myelofibrosis: a study on 359 patients of the AGIMM group. Am J Hematol. 2016;91(7):681-686.

30. Smalberg JH, Arends LR, Valla DC, Kiladjian JJ, Janssen HL, Leebeek FW. Myeloproliferative neoplasms in Budd-Chiari syndrome and portal vein thrombosis: a meta-analysis. Blood. 2012;120(25):4921-4928.

31. Stein BL, Saraf S, Sobol U, et al. Age-related differences in disease characteristics and clinical outcomes in polycythemia vera. Leuk Lymphoma. 2013;54(9):1989-1995.

32. Iurlo A, Cattaneo D, Gianelli U, Fermo E, Augello C, Cortelezzi A. Molecular analyses in the diagnosis of myeloproliferative neoplasmrelated splanchnic vein thrombosis. Ann Hematol. 2015;94(5):881-882.

33. Rumi E, Pietra D, Ferretti V, et al; Associazione Italiana per la Ricerca sul Cancro Gruppo Italiano Malattie Mieloproliferative Investigators. JAK2 or CALR mutation status defines subtypes of essential thrombocythemia with substantially different clinical course and outcomes. Blood. 2014; 123(10):1544-1551.

34. Rotunno G, Mannarelli C, Guglielmelli P, et al; Associazione Italiana per la Ricerca sul Cancro Gruppo Italiano Malattie Mieloproliferative Investigators. Impact of calreticulin mutations on clinical and hematological phenotype and outcome in essential thrombocythemia. Blood. 2014; 123(10):1552-1555

35. Barbui T, Finazzi G, Carobbio A, et al. Development and validation of an International Prognostic Score of thrombosis in World Health Organization-essential thrombocythemia (IPSET-thrombosis). Blood. 2012;120(26):5128-5133, quiz 5252.

36. Finazzi G, Carobbio A, Guglielmelli P, et al. Calreticulin mutation does not modify the IPSET score for predicting the risk of thrombosis among 1150 patients with essential thrombocythemia. Blood. 2014;124(16): 2611-2612. 
37. Pietra D, Rumi E, Ferretti VV, et al. Differential clinical effects of different mutation subtypes in CALR-mutant myeloproliferative neoplasms. Leukemia. 2016;30(2):431-438.

38. Tefferi A, Guglielmelli P, Larson DR, et al. Long-term survival and blast transformation in molecularly annotated essential thrombocythemia, polycythemia vera, and myelofibrosis. Blood. 2014;124(16):2507-2513, quiz 2615.

39. Elala YC, Lasho TL, Gangat N, et al. Calreticulin variant stratified driver mutational status and prognosis in essential thrombocythemia. Am J Hematol. 2016;91(5):503-506.

40. Tefferi A, Lasho TL, Huang J, et al. Low JAK2V617F allele burden in primary myelofibrosis, compared to either a higher allele burden or unmutated status, is associated with inferior overall and leukemia-free survival. Leukemia. 2008;22(4):756-761.

41. Kourie HR, Ameye L, Paesmans M, Bron D. Improved survival of calreticulin-mutated patients compared with Janus kinase 2 in primary myelofibrosis: a meta-analysis. Clin Lymphoma Myeloma Leuk. 2016; 16(5):264-268.

42. Grand FH, Hidalgo-Curtis CE, Ernst T, et al. Frequent CBL mutations associated with $11 \mathrm{q}$ acquired uniparental disomy in myeloproliferative neoplasms. Blood. 2009; 113(24):6182-6192.

43. Tefferi A, Lasho TL, Abdel-Wahab O, et al. IDH1 and IDH2 mutation studies in 1473 patients with chronic-, fibrotic- or blast-phase essential thrombocythemia, polycythemia vera or myelofibrosis. Leukemia. 2010; 24(7):1302-1309.

44. Oh ST, Simonds EF, Jones C, et al. Novel mutations in the inhibitory adaptor protein LNK drive JAK-STAT signaling in patients with myeloproliferative neoplasms. Blood. 2010;116(6):988-992.

45. Abdel-Wahab O, Mullally A, Hedvat C, et al. Genetic characterization of TET1, TET2, and TET3 alterations in myeloid malignancies. Blood. 2009;114(1):144-147.

46. Ernst T, Chase AJ, Score J, et al. Inactivating mutations of the histone methyltransferase gene EZH2 in myeloid disorders. Nat Genet. 2010; 42(8):722-726.

47. Carbuccia N, Murati A, Trouplin V, et al. Mutations of ASXL1 gene in myeloproliferative neoplasms. Leukemia. 2009;23(11):2183-2186.

48. Stein BL, Williams DM, O'Keefe C, et al. Disruption of the ASXL1 gene is frequent in primary, post-essential thrombocytosis and postpolycythemia vera myelofibrosis, but not essential thrombocytosis or polycythemia vera: analysis of molecular genetics and clinical phenotypes. Haematologica. 2011;96(10):1462-1469.

49. Lasho TL, Finke CM, Hanson CA, et al. SF3B1 mutations in primary myelofibrosis: clinical, histopathology and genetic correlates among 155 patients. Leukemia. 2012;26(5):1135-1137.

50. Lasho TL, Jimma T, Finke CM, et al. SRSF2 mutations in primary myelofibrosis: significant clustering with IDH mutations and independent association with inferior overall and leukemia-free survival. Blood. 2012; 120(20):4168-4171.

51. Ortmann CA, Kent DG, Nangalia J, et al. Effect of mutation order on myeloproliferative neoplasms. $N$ Engl J Med. 2015;372(7):601-612.
52. Tefferi A, Lasho TL, Finke C, et al. Targeted next-generation sequencing in polycythemia vera and essential thrombocythemia [abstract]. Blood. 2015:126(23). Abstract 354.

53. Vannucchi AM, Lasho TL, Guglielmelli P, et al. Mutations and prognosis in primary myelofibrosis. Leukemia. 2013;27(9):1861-1869.

54. Guglielmelli P, Lasho TL, Rotunno G, et al. The number of prognostically detrimental mutations and prognosis in primary myelofibrosis: an international study of 797 patients. Leukemia. 2014;28(9):1804-1810.

55. Lundberg P, Karow A, Nienhold R, et al. Clonal evolution and clinical correlates of somatic mutations in myeloproliferative neoplasms. Blood. 2014;123(14):2220-2228.

56. Tefferi A, Guglielmelli P, Lasho TL, et al. CALR and ASXL1 mutationsbased molecular prognostication in primary myelofibrosis: an international study of 570 patients. Leukemia. 2014;28(7):1494-1500.

57. Tefferi A, Guglielmelli P, Finke C, et al. Integration of mutations and karyotype towards a genetics-based Prognostic Scoring System (GPSS) for primary myelofibrosis. Blood. 2014;124(21):406.

58. Vannucchi AM, Guglielmelli P, Rotunno G, et al. Mutation-enhanced International Prognostic Scoring System (MIPSS) for primary myelofibrosis: an AGIMM \& IWG-MRT Project. Blood. 2014;124(21):405.

59. Devlin R, Gupta V. Myelofibrosis: to transplant or not to transplant? Hematology Am Soc Hematol Educ Program. 2016;2016:543-551.

60. Quintás-Cardama A, Abdel-Wahab O, Manshouri T, et al. Molecular analysis of patients with polycythemia vera or essential thrombocythemia receiving pegylated interferon $\alpha-2$ a. Blood. 2013;122(6):893-901.

61. Kuriakose E, Vandris K, Wang YL, et al. Decrease in JAK2 V617F allele burden is not a prerequisite to clinical response in patients with polycythemia vera. Haematologica. 2012;97(4):538-542.

62. Verger E, Cassinat B, Chauveau A, et al. Clinical and molecular response to interferon- $\alpha$ therapy in essential thrombocythemia patients with CALR mutations. Blood. 2015;126(24):2585-2591.

63. Baerlocher GM, Burington B, Snyder DS. Telomerase inhibitor imetelstat in essential thrombocythemia and myelofibrosis. N Engl J Med. 2015; 373(26):2580

64. Verstovsek S, Mesa RA, Gotlib J, et al. A double-blind, placebocontrolled trial of ruxolitinib for myelofibrosis. N Engl J Med. 2012; 366(9):799-807.

65. Harrison C, Kiladjian JJ, Al-Ali HK, et al. JAK inhibition with ruxolitinib versus best available therapy for myelofibrosis. N Engl J Med. 2012; 366(9):787-798.

66. Passamonti F, Caramazza D, Maffioli M. JAK inhibitor in CALR-mutant myelofibrosis. N Engl J Med. 2014;370(12):1168-1169.

67. Rampal R, Al-Shahrour F, Abdel-Wahab O, et al. Integrated genomic analysis illustrates the central role of JAK-STAT pathway activation in myeloproliferative neoplasm pathogenesis. Blood. 2014;123(22):e123-e133.

68. Patel KP, Newberry KJ, Luthra R, et al. Correlation of mutation profile and response in patients with myelofibrosis treated with ruxolitinib. Blood. 2015;126(6):790-797.

69. Tefferi A, Lasho TL, Begna KH, et al. A pilot study of the telomerase inhibitor imetelstat for myelofibrosis. N Engl J Med. 2015;373(10):908-919. 